\title{
DEPENDENCE OF P-BAND INTERFEROMETRIC HEIGHT ON FOREST PARAMETERS FROM SIMULATION AND OBSERVATION
}

\author{
$M$ Lavalle $^{(1)}, M L_{\text {Williams }}^{(2)}$, S Hensley $^{(3)}$, E Pottier $^{(4)}$ and D Solimini $^{(1)}$ \\ ${ }^{(1),(4)}$ Tor Vergata University, Via del Politecnico 1, 00133 Rome, Italy \\ ${ }^{(1)}$ marcolavalle@gmail.com \\ ${ }^{(2)}$ Fugro-EarthData, 7320 Executive Way, Frederick, MD 21704, USA. \\ ${ }^{(2)}$ mlwilliams@earthdata.com \\ (3) Jet Propulsion Laboratory, California Institute of Technology, 4800 Oak Grove Drive, Pasadena, CA 91109, USA. \\ ${ }^{(4)}$ University of Rennes 1, IETR, UMR CNRS 6164, 263 Avenue Général Leclerc, CS 74205, \\ 35042 Rennes Cedex, France.
}

\begin{abstract}
GeoSAR is a unique dual-band, interferometric SAR (DBInSAR) sensor capable of collecting single-pass, Xband (VV) and P-band (HH) interferometric data simultaneously. In this paper we examine the dependence of the P-band $\mathrm{HH}$ interferometric phase centre height upon forest and terrain parameters. We develop a simple model for P-band GeoSAR observations, and use the model to show how the elevation in P-band HH phase centre height above true ground height is related to the volume-to-ground scattering ratio. GeoSAR is not fully-polarimetric, but records cross-polar (HV) returns at P-band (although not interferometrically). We conjecture that these returns are dominated by direct-volume scattering and related to the direct-volume HH backscatter. We use this relationship to model the dependence of the P-band HH DTM height upon the $\mathrm{HV} / \mathrm{HH}$ ratio, and the difference in X-band DEM with P-band DTM heights. The relationships are examined using simulated forest InSAR data, and a model is proposed for ground-height and tree-height estimation using DBInSAR that does not require full polarimetry.
\end{abstract}

\section{INTRODUCTION}

Scattering of electromagnetic waves from vegetation is strongly dependent on frequency. At X-band, scattering is predominantly "first-surface", and, in general, the X-band VV interferometric phase centre is anticipated to be close to the top of vegetation canopies. At lower frequencies, such as P-band, $\mathrm{HH}$ returns are, in general, more strongly influenced by ground-volume interactions, and the P-band HH phase centre is expected to lie closer to the ground. Thus the difference between the X-band VV digital surface map (DSM) height and the P-band HH digital terrain map (DTM) height is related to the height of the vegetation. This "surrogate" vegetation height has been used in the retrieval of biomass for areas of tropical forest [1]. The GeoSAR dual-frequency, interferometric SAR was developed for wide-area, airborne mapping applications by NASA's Jet Propulsion Laboratory [2] and is now operated commercially by Fugro-EarthData on a Gulfstream II jet aircraft. GeoSAR collects X-band (VV, 9.7GHz) and Pband (HH, 0.35GHZ) interferometric data in single-passes, from which are derived digital elevation models. The combination of wide-area mapping capability and sensitivity to forest height make GeoSAR an invaluable tool for the large-scale estimation and monitoring of above-ground carbon stocks.

Both evidence and theory suggest that volume scattering effects will lift the P-band $\mathrm{HH}$ phase centre off the ground somewhat, even though the ground-volume scattering is strong. An example of a raised P-band $\mathrm{HH}$ phase centre under forest is given below in Figures 1 and 2.
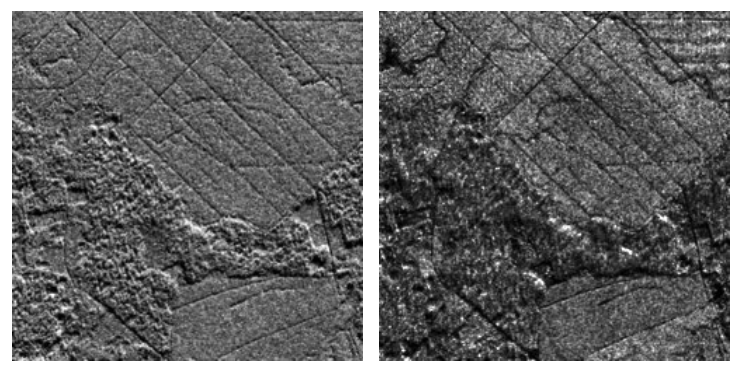

Figure 1. X-band (left) and P-band (right) images of an area containing tropical forest and cultivation.

The figures show a forested area next to a cultivated area distinguished most clearly in the P-band magnitude data. The edge of the cultivated area is evident in both the X-band DSM (left) and the P-band DTM (right). Although no ground data were available for this area, the evidence appears to suggest a slight rise in P-band DTM height below the neighboring forest canopy of a few meters. That the forest canopy is approx. $20-25 \mathrm{~m}$ tall can be deduced from the X-P height difference. 

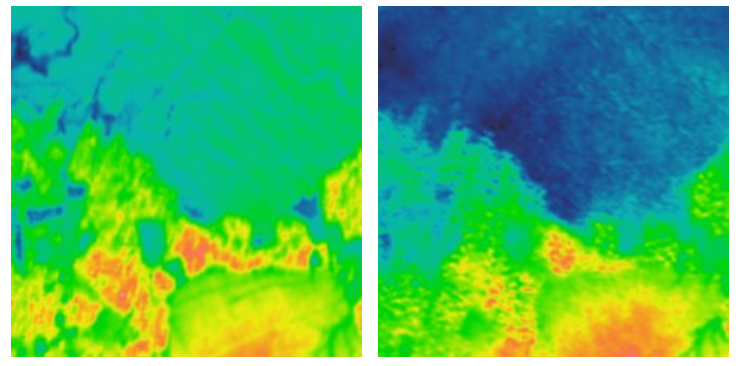

Figure 2. X-band DSM (left) and P-band DTM (right) of the same area in Figure 1. Note DEM color scales differ between bands.

In order to better exploit the surrogate vegetation height measurement available from GeoSAR observations we need to determine the relationship between our usual understanding of tree height, and the X-band - P-band interferometric height difference. In part this requires an understanding of where the P-band height is above the ground under forest canopies.

\section{THEORETICAL MODEL}

We begin by considering the "random-volume-overground" [3] model for interferometric coherence in our single P-HH channel for a canopy of height $h$, for heights $z_{g} \leq z \leq h+z_{g}$ :

$$
\hat{\gamma}=\gamma e^{i \phi_{\gamma}}=\int_{z_{g}}^{z_{g}+h} \sigma(z) e^{i k z} d z / \int_{z_{g}}^{z_{g}+h} \sigma(z) d z
$$

where $\kappa$ is the interferometric wavenumber. We make the approximation that the total backscattering coefficient is the sum of direct-ground $(d g)$, direct-volume $(d v)$ and groundvolume $(g v)$ terms and treat the $d g$ term as not significant. The total P-HH backscattering coefficient is

$$
\sigma(z) \cong e^{-4 k k_{2} h}\left[\int_{0}^{h} n(y) \sigma_{g v}\left(y+z_{g}\right) d y+\int_{0}^{h} e^{4 k, y} n(y) \sigma_{d v}\left(y+z_{g}\right) d y\right]
$$

where $y=z-z_{g}$ is the height above ground-level, $n(y)$ is the scatterer density per unit depth at height $y, k_{z}$ is the imaginary part of the z-component of the wavenumber in the canopy, and $\sigma_{a b}\left(y+z_{g}\right)$ is the brightness per unit height of scatterers at height $y$. Two separate approximations for scattering as a function of height in the canopy lead to similar but different models for coherence. The first suggests that

$$
\bar{\rho} \bar{\sigma}_{a b}=e^{4 k_{z} y} n(y) \sigma_{a b}\left(y+z_{g}\right)=\text { constant }
$$

whilst the alternative has

$$
\bar{\rho} \bar{\sigma}_{d v}=n(y) \sigma_{d v}\left(y+z_{g}\right)=\text { constant } .
$$

In the first model the attenuated scattering is constant with depth, and in the second the un-attenuated scattering is constant with depth. Using first (1), (2) and (3), and then (1), (2) and (4) we obtain expressions for P-HH interferometric coherence at low frequency in each approximation as:

$$
\hat{\gamma}=\gamma e^{i \phi_{\gamma}} \cong e^{i \kappa z_{g}} \frac{1+\eta e^{i \kappa h / 2} \operatorname{Sinc}(\kappa h / 2)}{1+\eta}
$$

and

$$
\hat{\gamma}=\gamma e^{i \phi_{\gamma}} \cong e^{i \alpha_{g}} \frac{1+\eta e^{i k \hbar t}}{1+\eta}
$$

In both cases $\eta=\sigma_{d v} / \sigma_{g v}$ is the ratio of $d v$ to $g v$ scattering for the P-HH channel. In both cases the models suggest that as $\eta$ increases the ground phase increases, and the $\mathrm{P}-\mathrm{HH}$ phase centre rises above the ground level. Figure 3 illustrates this effect for typical GeoSAR imaging parameters and a $25 \mathrm{~m}$ tall canopy:

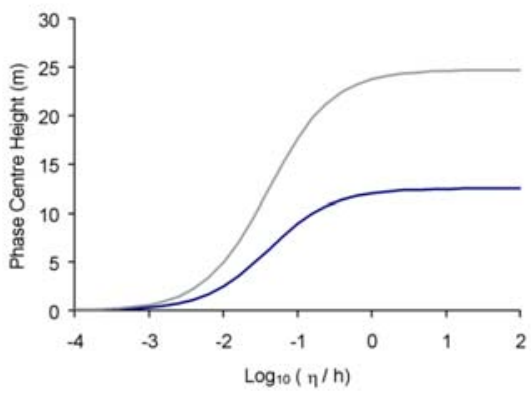

Figure 3. Theoretical variations of the $\mathrm{PHH}$ phase centre with $d v / g v$ ratio. At $\mathrm{P}$-band $\eta / h \approx 0.01$ and the $\mathrm{P}-\mathrm{HH}$ phase centre sits a meter or so above the surface. The effect is much greater at Lband. Blue curve corresponds to (5), light grey curve corresponds to (6).

Equations (5) and (6) are each two equations: one for coherence magnitude, the other for coherence phase. This suggests that a height correction to ground could be found from GeoSAR measurements by approximating $h$ as the difference between the X-VV and P-HH height differences, then estimating $\eta$ from $\mathrm{P}-\mathrm{HH}$ coherence magnitude, and finally a phase correction from $\mathrm{P}-\mathrm{HH}$ coherence phase. Unfortunately wavelength and baseline dimensions conspire against GeoSAR and P-HH coherence is quite insensitive to $d v / g v$ ratio: making it an ideal P-band mapping instrument! 


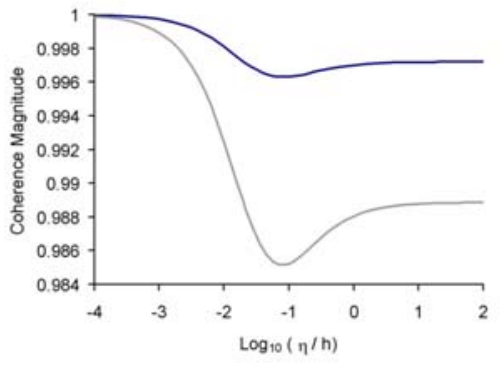

Figure 4. GeoSAR P-HH coherence magnitude variations as a function of $d v / g v$ ratio for a $25 \mathrm{~m}$ canopy. Variation is insufficient in all modes and approximations to be detectible.

However all is not lost if we recall that we also measure $\mathrm{P}-\mathrm{HV}$ (although not interferometrically). If we assume that total $\mathrm{P}-\mathrm{HV}$ is equivalent to $d v \mathrm{P}-\mathrm{HV}$, and further that $d v \mathrm{P}-$ $\mathrm{HH}$ is proportional to $d v \mathrm{P}-\mathrm{HV}$, then we can use the $\mathrm{P}$ $\mathrm{HV} / \mathrm{P}-\mathrm{HH}$ ratio to estimate the $d v / g v$ ratio [4]:

$$
\eta=\frac{1}{\frac{\sigma_{H H}}{h \alpha_{c} \sigma_{H V}}-1}
$$

where we have assumed that $d v \mathrm{P}-\mathrm{HH}$ is related to $d v \mathrm{P}-\mathrm{HV}$ through the factor $h \alpha_{c}$, where $\alpha_{c}$ is constant that depends only on the tree species.

Now we may calculate height corrections to ground using the $\mathrm{X}-\mathrm{VV}$ and $\mathrm{P}-\mathrm{HH}$ heights, and $\eta=\sigma_{d v} / \sigma_{g v}$, according to our previous models as:

$$
\delta h=\frac{1}{\kappa} \tan ^{-1}\left\{\frac{\eta \operatorname{Sinc}\left(\phi_{h}\right) \sin \phi_{h}}{1+\eta \operatorname{Sinc}\left(\phi_{h}\right) \cos \phi_{h}}\right\}
$$

and

$$
\delta h=\frac{1}{\kappa} \tan ^{-1}\left\{\frac{\eta \sin 2 \phi_{h}}{1+\eta \cos 2 \phi_{h}}\right\}
$$

wherein $\phi_{h}=\kappa h / 2$ and $\phi_{\gamma}=\phi_{z}+\delta \phi=\phi_{z}+\delta h / \kappa$. Thus for a $25 \mathrm{~m}$ tall canopy with $\eta / h \approx 0.01 \mathrm{~m}^{-1}$, the height error is approximately $2.5 \mathrm{~m}$ with the first model and $5.0 \mathrm{~m}$ with the second, which is consistent with our observations. In what follows we use a coherent, forest SAR simulation (PolSARproSim [5]) to perform calculations at P-band to test the validity of these theoretical models.

\section{SAR SIMULATION}

A set of SAR simulations has been performed to test the assumptions discussed in Sec. 2, to assess the value of $\alpha_{c}$ in (7), and to validate the inversion procedure outlined in Sec.
4. We have conducted 26 coherent simulations of Pine tree forest using PolSARProSIM. The set of simulations has been obtained by increasing the forest height from $5 \mathrm{~m}$ up to $30 \mathrm{~m}$. A typical GeoSAR acquisition geometry has been assumed (Table 1) which leads to a vertical wavenumber $\kappa$ $\approx 0.2 \mathrm{~m}^{-1}$ and to an ambiguity height $h_{a} \approx 31.4 \mathrm{~m}$.

\begin{tabular}{|l|c|}
\hline Parameter & Value \\
\hline Sensor altitude & $1.0 \times 10^{4} \mathrm{~m}$ \\
\hline Incident angle & $45 \mathrm{deg}$ \\
\hline Central frequency & $1.3 \times 10^{9} \mathrm{~Hz}$ \\
\hline Azimuth resolution & $3.53 \mathrm{~m}$ \\
\hline Range resolution & $2.5 \mathrm{~m}$ \\
\hline Horizontal baseline & $20 \mathrm{~m}$ \\
\hline Vertical baseline & $0 \mathrm{~m}$ \\
\hline
\end{tabular}

Table 1 Sensor and acquisition geometry characteristics used in the Pine tree simulations for the validation of the model outlined in Sec. 2 .

The importance of SAR simulation using PolSARproSIM lies on the ability to simulate separately the effects of the ground and the effects of the canopy at different polarizations. In our specific case, we are interested in simulating the total return, the direct-vegetation and groundvegetation return in the $\mathrm{HH}$ - and HV-channel. Figure 4 shows the individual scattering mechanisms used for the analysis, i.e. the $d v \mathrm{P}-\mathrm{HV}, d v \mathrm{P}-\mathrm{HH}$, total P-HV, total P-HV and $g v \mathrm{P}-\mathrm{HH}$.

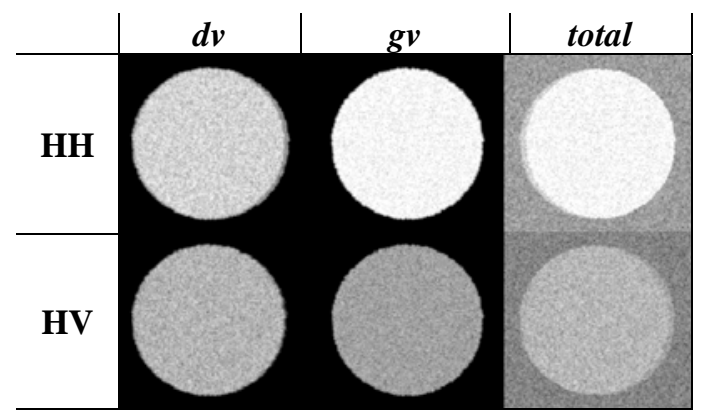

Figure 4 Example of PolSARProSIM simulations and individual scattering mechanisms (master images). Simulations are obtained using GeoSAR imaging geometry and a $20 \mathrm{~m}$ tall Pine forest.

The factor $\alpha_{c}$ is estimated from the central part of the images in Figure 4, using both the definition and the approximation

$$
\alpha_{c}=\frac{\sigma_{H H}^{(d v)}}{h \sigma_{H V}^{(d v)}} \cong \frac{\sigma_{H H}^{(d v)}}{\left(h-\delta h_{\text {int }}\right) \sigma_{H V}}
$$

where $h$ is the outset height of the simulation and $\delta h_{\text {int }}$ is the height of the P-HH interferometric phase center. The 
simulated difference $h_{X P}=h-\delta h_{\text {int }}$ corresponds to the Xband - P-band interferometric height difference obtained from real data, since the X-band phase center is located at the top of canopy $(h)$ the P-band phase center can be estimated by interferometric processing $\left(\delta h_{\text {int }}\right)$. Figure 5 shows the comparison between the two estimates of $\alpha_{c}$ and confirms that the two curves are very close, especially for trees taller than $10 \mathrm{~m}$. The average value of $\alpha_{c}$ that can be assumed for pine tree is about 0.45 . The second parameter that we test is the volume-to-ground scattering ratio. Again, the $d v / g v$ ratio can be estimated from simulation through its definition or the approximation (7). In Figure 6 we report the trend of $\eta$ for the two alternative estimates. The curves proof that our approximated expression holds, especially for trees taller than $10 \mathrm{~m}$.
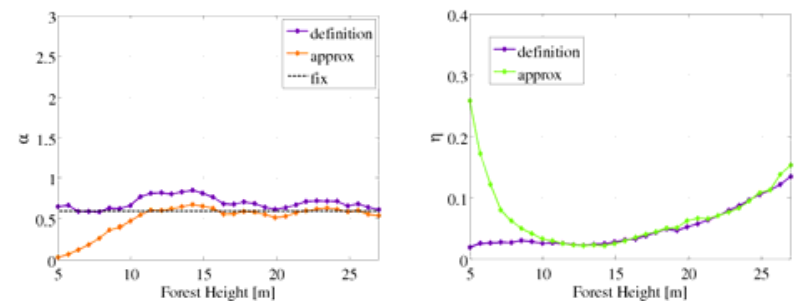

Figure 5 Validation of the alpha parameter (left) and of the approximated volume-to-ground ratio (right).

\section{INVERSION PROCEDURE AND RESULTS}

The previous analysis ensures that the models of Sec. 2 can be applied to correct the $\mathrm{P}-\mathrm{HH}$ interferometric phase center height and to obtain a better estimate of forest height using GeoSAR acquisitions. In this section we provide a clear procedure and compare the performance of the two models. The following procedure is proposed.

1. Estimate the canopy depth using the X-band - P-band DEM height difference $h_{X P}$.

2. Estimate the volume-to-ground ratio $\eta$ from $\mathrm{P}-\mathrm{HH}$ and P-HV measurements, using (7) and $h_{X P}$.

3. Estimate the unwrapped ground phase $\delta h$ using $\eta$ and $h_{X P}$ from the model equation (8) or (9).

4. Correct the height estimate $h_{X P}$ with the height shift $\delta h$ and iterate the procedure if necessary.

This procedure has been tested using PolSARProSIM simulated data. Figure 7 shows that both models perform well in the estimation of the P-HH phase shift. In the case of model I, $\delta h$ is underestimated. On the contrary, using model II $\delta h$ is slightly overestimated. This suggests that, for pine trees, the extinction along the vertical dimension is between the uniform profile and the exponential profile. The procedure may be refined by including a correction coefficient that accounts for terrain slope in the $\sigma_{H V}$ measurements. PolSARProSIM allows to asses this coefficient and this will be shown in a future stage of our work.

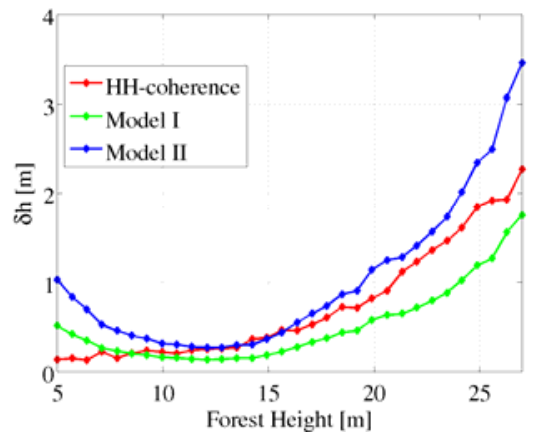

Figure 7 Comparison between P-HH phase center heights from interferometric processing and from model equations. Plots are generated using PolSARProSIM simulations.

\section{CONCLUSIONS}

We have shown that, using a DBInSAR system that acquires at X-band and $\mathrm{P}$-band, it is possible to estimate the tree height of a forested area without using full-pol data. We have presented a new approach based on the RVoG model. The validity of the assumptions made in our approach has been tested using PolSARProSIM Pine forest simulations. The retrieval procedure, finally, has been successfully applied to simulated data.

\section{REFERENCES}

[1] T Neeff et al., Tropical Forest Biomass Measurement by Backscatter and DEM Information as Derived from Airborne SAR, Proceedings of the International Geoscience and Remote Sensing Symposium, 2003.

[2] A K Wheeler, and S Hensley, The GeoSAR airborne mapping system, Record of the IEEE 2000 International Radar Conference, 2000, Page(s):831 - 835.

[3] R N Treuhaft, S N Madsen, M Moghaddam, and J J van Zyl. Vegetation characteristics and underlying topography from interferometric radar. Radio Science, 31(6):1449-1485, 1996.

[4] M L Williams, A Model for P-band Phase Height Estimation, Fugro-EarthData, Private Communication, $18^{\text {th }}$ December, 2008.

[5] M.L. Williams et al., Forest Coherent SAR Simulation within PolSARPro: an Educational Toolbox for PolSAR and PolInSAR Data Processing, Asian Conference on Remote Sensing, Kuala Lumpur, Malaysia, 2007. 